\title{
Optimal pacing modes after cardiac transplantation: is synchronisation of recipient and donor atria beneficial?
}

\author{
Gareth Parry, Katie Malbut, John H Dark, Rodney S Bexton
}

\begin{abstract}
Objective-To investigate the response of the transplanted heart to different pacing modes and to synchronisation of the recipient and donor atria in terms of cardiac output at rest.

Design-Doppler derived cardiac output measurements at three pacing rates $(90 / \mathrm{min}, 110 / \mathrm{min}$ and $130 / \mathrm{min})$ in five pacing modes: right ventricular pacing, donor atrial pacing, recipient-donor synchronous pacing, donor atrial-ventricular sequential pacing, and synchronous recipient-donor atrial-ventricular sequential pacing.

Patients-11 healthy cardiac transplant recipients with three pairs of epicardial leads inserted at transplantation.

Results-Donor atrial pacing $(+11 \%$ overall) and donor atrial-ventricular sequential pacing $(+8 \%$ overall) were significantly better than right ventricular pacing ( $p<0.001)$ at all pacing rates. Synchronised pacing of recipient and donor atrial segments did not confer additional benefit in either atrial or atrial-ventricular sequential modes of pacing in terms of cardiac output at rest at these fixed rates.

Conclusions-Atrial pacing or atrialventricular sequential pacing appear to be appropriate modes in cardiac transplant recipients. Synchronisation of recipient and donor atrial segments in this study produced no additional benefit. Chronotropic competence in these patients may, however, result in improved exercise capacity and deserves further investigation.
\end{abstract}

\section{Departments of}

Cardiology and

Cardiopulmonary

Transplantation,

Freeman Hospital,

Newcastle upon Tyne

G Parry

K Malbut

JH Dark

R S Bexton

Correspondence to

Dr Gareth Parry,

Department of Cardiology,

Freeman Hospital,

Freeman Hospital, NE7 7DN.

Accepted for publication 5 March 1992

\section{(Br Heart J 1992;68:195-8)}

Permanent pacemaker insertion may be necessary after cardiac transplantation for the treatment of sinus node dysfunction or complete heart block. Rates of pacemaker implantation vary in reported series from $4 \%-24 \% .{ }^{1-3}$ Our data suggest that only those patients with sinus node dysfunction will require long-term pacing, and these may comprise fewer than $2 \%$ of cardiac transplant recipients. ${ }^{2}$ Although the number of patients needing long-term pacing may thus be small, for those who do need pacing the optimal mode should be used to realise the maximum potential of the transplanted heart. Although physiological pacing has been shown to be haemodynamically better than ventricular pacing in the native heart, ${ }^{4-6}$ this may not necessarily be the case after transplantation.

The surgical technique of orthotopic cardiac transplantation has changed little since first described by Cass and Brock in 1959.7 Donor atria are sutured to recipient atrial remnants to form a pair of composite atria, the two components of each composite atrium being electrically isolated. Thus donor and recipient atrial systole are independent with resultant incoordinate atrial contraction. Few data are available on the haemodynamic contribution of atrial contraction to ventricular filling in transplanted hearts. We have noted a decreased active atrial contribution to left ventricular filling in transplant recipients compared with normal subjects (data submitted for publication) and others have shown an increase in peak Doppler transmitral velocity in late diastole with coincidental recipient and donor atrial systole. ${ }^{8}$ These findings suggest altered left ventricular filling after cardiac transplantation; left ventricular output (that is, cardiac output) may thus not reach its full potential.

Physiological pacing is recommended for many patients with native hearts. ${ }^{9}$ The benefits are twofold: improved exercise tolerance compared with ventricular pacing, ${ }^{10}$ and reduced likelihood of pacemaker syndrome. ${ }^{11}$ Although the second may also be true in transplant recipients, pacing one part of the atrium (donor) may not be more beneficial in terms of improved cardiac function in the transplanted heart than ventricular pacing.

Coordination of the atrial components by electrical connection is theoretically attractive and has been described in one patient. ${ }^{12}$ As well as atrial synchrony, another theoretical benefit of electrical atrial connection is chronotropic competence in the transplanted heart in response to neural stimuli to the recipient sinus node.

The aims of this study were (a) to investigate conventional pacing modes in the transplanted heart, and $(b)$ to compare conventional physiological pacing with synchronous recipient-donor atrial physiological pacing. 


\section{Patients and methods}

After ethical approval at our hospital, all cardiac transplant recipients had three pairs of epicardial pacing wires inserted at transplantation-to recipient right atrium, donor right atrium and right ventricle. Subsequent study of these patients was dependent on several criteria. Patients had to be $(a)$ well and able to give informed consent, $(b)$ not on inotropes, (c) within 48 hours of a normal cardiac biopsy, and $(d)$ in sinus rhythm with normal atrioventricular conduction.

\section{CARDIAC PACING}

Epicardial cardiac pacing was performed through an external dual chamber pacing generator. Patients were paced in a random sequence in each of five modes where possible -namely, ventricular pacing alone, donor atrial pacing, synchronous recipient-donor atrial pacing, conventional donor atrial and sequential ventricular pacing, and synchronous recipient-donor atrial and sequential ventricular pacing. Three pacing rates were used for our investigation-90 per minute, 110 per minute, and 130 per minute. As heart transplant recipients have a high basal heart rate (due to denervation), 90 per minute was chosen as our basal rate, with 110 and 130 per minute to reflect rates expected in normal subjects with exercise. For AV sequential pacing, the AV delay was set at 200 ms unless normal conduction occurred necessitating a shorter delay. In an individual patient this delay was maintained constant at all pacing rates and in both $\mathrm{AV}$ sequential modes.

\section{DOPPLER MEASUREMENT OF CARDIAC OUTPUT}

Cardiac output was determined noninvasively by a previously validated technique by Doppler echocardiography. ${ }^{13}$ Continuous wave Doppler waveforms were obtained from the ascending aorta with Hewlett-Packard echocardiographic equipment. Flow velocity integrals were found by two observers who traced from the baseline around the velocity curve with a digitising tablet linked to a microcomputer. ${ }^{14}$ Ten waveforms were measured blindly in each mode and at each pacing rate. Aortic valve dimensions were measured with cross sectional echocardiography with cardiac output derived by the formula:

cardiac output $=$ stroke distance $\times$ heart

(pacing) rate

(where stroke distance $=$ aortic valve area $\times$

Doppler velocity integral).

Statistical analysis was by paired $t$ tests.
Results

\section{PATIENTS}

Of 21 consecutive patients who had three pairs of epicardial pacing wires placed at transplantation, only 11 met our inclusion criteria for further study. These 11 patients $(10 \mathrm{men})$ were investigated within 21 days of transplantation. The mean age of the recipients was 45 (range 18-53), with a mean donor age of 27 (range 1547). All patients were in sinus rhythm with right bundle branch block (four patients) or had an RSR' $^{\prime}$ pattern with a QRS duration within normal limits (seven patients). Two patients had resting heart rates above 90 per minute during part or all of the pacing study. Pacing was not possible in all pacing modes in all patients because of various difficulties including basal rates above 90 per minute, induction of extrasystoles, and failure to capture, especially with synchronous atrial pacing. Thus of 165 possible combinations in our 11 patients, 49 were not carried out. The results are presented according to the number of patients in whom comparisons were possible, and hence the mean values for cardiac output in the same pacing mode between these comparisons differ. To obviate statistical problems with small numbers, and to confirm that the individual differences between pacing modes at different pacing rates were correct, we have averaged all measurements from the different rates obtained in a pacing mode to obtain an overall mean value for cardiac output in that mode. This allows statistical comparison between the pacing modes.

\section{CONVENTIONAL PACING MODES}

Both donor right atrial pacing and conventional AV sequential pacing were better than right ventricular pacing in our patients (table 1 ). The increase in cardiac output found with donor right atrial pacing compared with right ventricular pacing was $+13 \%$ at 90 per minute, $+10 \%$ at 110 per minute, and $+11 \%$ at 130 per minute. Overall from 27 comparisons, the benefit was $+11 \%$. The increase in cardiac output with conventional AV sequential pacing compared with right ventricular pacing was $+9 \%,+7 \%$, and $+8 \%$ respectively at the pacing rates studied, and $+8 \%$ overall from 24 comparisons. The difference between donor atrial pacing and conventional $\mathrm{AV}$ sequential pacing was small at all rates $(2 \cdot 85 \%$ to $3.30 \%$ in favour of donor atrial pacing), but this was not significant even with overall means from 24 observations.

Table 1 Comparison of conventional pacing modes

\begin{tabular}{|c|c|c|c|c|c|c|c|c|c|}
\hline & $D A$ & $R V$ & & $D-V$ & $R V$ & & $D A$ & $D-V$ & \\
\hline $\begin{array}{c}\text { Pacing rate } \\
90 / \mathrm{min} \\
110 / \mathrm{min} \\
130 / \mathrm{min}\end{array}$ & $\begin{array}{l}7 \cdot 30(1 \cdot 80) \\
8 \cdot 28(2 \cdot 04) \\
9 \cdot 45(2 \cdot 57)\end{array}$ & $\begin{array}{l}6.46(1.70) \\
7.54(2.15) \\
8.54(2.51)\end{array}$ & $\begin{array}{l}p<0.02(n=8) \\
p<0.02(n=9) \\
p<0.05(n=10)\end{array}$ & $\begin{array}{l}6 \cdot 80(1.43) \\
8 \cdot 08(2 \cdot 22) \\
8.85(2.98)\end{array}$ & $\begin{array}{l}6 \cdot 24(1.53) \\
7.54(2.30) \\
8 \cdot 22(2.62)\end{array}$ & $\begin{array}{l}p<0.01(n=8) \\
p<0.01(n=8) \\
p<0.02(n=8)\end{array}$ & $\begin{array}{l}6.93(1.59) \\
8.31(2.03) \\
9 \cdot 14(2.39)\end{array}$ & $\begin{array}{l}6.71(1.52) \\
8.08(2.08) \\
8.85(2.98)\end{array}$ & $\begin{array}{l}\text { NS }(n=7) \\
\text { NS }(n=9) \\
\text { NS }(n=8)\end{array}$ \\
\hline Mean & $8 \cdot 42(2 \cdot 29)$ & $7 \cdot 59(2 \cdot 26)$ & $p<0.001(n=27)$ & $7.91(2.36)$ & $7 \cdot 33(2 \cdot 26)$ & $p<0.001(n=24)$ & $8 \cdot 19(2 \cdot 15)$ & $7.93(2.36)$ & NS $(n=24$ \\
\hline
\end{tabular}

Values for cardiac output (litres per minute) mean (SD); DA, donor atrial pacing; D-V, donor atrial-ventricular sequential pacing; mean, mean for all patients for all pacing rates; $R V$, right ventricular pacing. 
Table 2 Comparison of donor atrial pacing with synchronous recipient-donor atrial pacing alone, and with sequential ventricular pacing

\begin{tabular}{|c|c|c|c|c|c|c|}
\hline & $D A$ & $R D A$ & & $D-V$ & $R D-V$ & \\
\hline $\begin{array}{c}\text { Pacing rate } \\
90 / \mathrm{min} \\
110 / \mathrm{min} \\
130 / \mathrm{min}\end{array}$ & $\begin{aligned} 8.15 & (1.38) \\
9.06 & (1.60) \\
10.87 & (1.81)\end{aligned}$ & $\begin{aligned} 8.53 & (1.62) \\
8.98 & (1.26) \\
10.46 & (1.50)\end{aligned}$ & $\begin{array}{l}\text { NS }(n=4) \\
\text { NS }(n=6) \\
\text { NS }(n=6)\end{array}$ & $\begin{array}{r}7.54(1.54) \\
9.10(1.64) \\
10.80(2.40)\end{array}$ & $\begin{aligned} 7.79 & (1.52) \\
9.37 & (1.35) \\
10.75 & (1.99)\end{aligned}$ & $\begin{array}{l}\text { NS }(n=2) \\
\text { NS }(n=5) \\
\text { NS }(n=4)\end{array}$ \\
\hline Mean & $9.40(1.96)$ & $9.32(1.64)$ & NS $(n=16)$ & $9.43(2.14)$ & $9.58(1.84)$ & $N S(n=11)$ \\
\hline
\end{tabular}

Footnotes as for table 1.

RDA, recipient-donor synchronous atrial pacing; $R D-V$, recipient-donor synchronous atrial-ventricular sequential pacing.

\section{SYNCHRONOUS RECIPIENT-DONOR ATRIAL} PACING

As mentioned already, to obviate any statistical problems associated with small numbers we have averaged the results in each mode of pacing. Despite this, there was no difference between donor and recipient-donor modes of pacing in our patients (table 2).

\section{SYNCHRONOUS RECIPIENT-DONOR ATRIAL AV} SEQUENTIAL PACING

As with atrial pacing, there was no statistical difference between conventional AV sequential pacing and synchronous recipient-donor atrial AV sequential pacing (table 2 ).

\section{Discussion}

We have shown that physiological pacing is indeed better than ventricular pacing alone in cardiac transplant recipients. Our data agree with those of an invasive study by Midei $e t$ al who reported a $19 \%$ mean improvement in cardiac output with atrial pacing at a single rate in nine patients. ${ }^{15}$ Our theoretical supposition that the benefit of physiological pacing would not be as great as in native hearts is supported to some degree by the percentage benefit seen in our patients compared with the benefit reported in patients with native hearts. In our study conventional AV sequential pacing improved cardiac output by $8 \%$ overall compared with ventricular pacing; in native hearts, a similar study reported a benefit of $27 \%$ at a paced rate of 70 per minute. ${ }^{4}$ Possible reasons for this lower benefit in cardiac transplant recipients include asynergy of recipient and donor atrial segments, but we were unable to show a benefit from synchronous electrical activation of the two atrial segments in terms of cardiac output at rest at the pacing rates studied. Denervation of the transplanted heart is unlikely to result in a decreased force of contraction of the donor atrium as the absence of parasympathetic stimulation should relieve the atrium of its normally negatively inotropic effects. ${ }^{16}$ Another possible cause may be damage to the atrium during surgery, or distortion of the donor atrium after surgery resulting in incoordination of donor atrial contraction.

Why did electrical synchrony of recipient and donor atria not produce a benefit? In our apparently healthy cardiac transplant recipients it is possible that atrial synchrony was unnecessary to achieve maximal cardiac output at rest. After episodes of acute rejection, however, when left ventricular compliance may be reduced, a stronger active atrial component to left ventricular filling may become vital to preserve left ventricular function. The function of atrial contraction in normal subjects is to enhance ventricular filling above that which is possible by passive filling alone at normal mean atrial pressures. To preserve ventricular filling in the absence of atrial contraction, atrial pressure during diastole must therefore be higher than in a heart with normal anatomy. A decrease in left ventricular compliance will necessitate a further rise in mean atrial pressure which may precipitate pulmonary oedema. Thus electrical synchrony may be a prophylactic measure to avoid this sequence of events which may occur during rejection-either acutely or chronically.

We readily acknowledge that the numbers in our study are small, in particular those patients in whom recipient-donor atrial pacing was possible. Even summation of our data at all pacing rates in each mode to enable statistical comparison of larger numbers, however, did not suggest that an important benefit exists overall in transplant recipients from synchronous recipient-donor atrial pacing. We therefore stopped further enrolment in this study.

The cardiac output values found seem high when compared with reported cardiac output values from normal subjects at rest. The basal cardiac outputs in this study were only slightly higher and did not differ significantly from that seen in a previous study of cardiac transplant patients performed here. ${ }^{17} \mathrm{~A}$ possible explanation for the high cardiac outputs seen in our study, however, is that all patients were within 21 days of major surgery and thus likely to be still in a hypermetabolic state. Another explanation might be that many of our patients were large in physical size (with body surface areas in excess of $2 \mathrm{sq} \mathrm{m}$ ), and perhaps cardiac index would be more appropriate for absolute values. Our main objective in this study was not to provide absolute values for cardiac output in transplant recipients, but rather to emphasise the difference seen in cardiac output with different pacing modes.

Our study was performed at rest, and our results may not necessarily correlate with differences in cardiac output found during exercise at the pacing rates studied in the various pacing modes. We have no reason to suppose that there would be more benefit from ventricular pacing during exercise than with atrial pacing. Likewise, exercise is unlikely to result in any major haemodynamic change such that 
synchrony of recipient and donor atria would be significantly better.

Chronotropic competence during exercise may be beneficial in the transplanted heart, however, as the chronotropic response to exercise is abnormal in all cardiac transplant recipients. ${ }^{1819}$ Electrical connection of the atria would bring the donor under the control of the recipient sinus node (at least at heart rates above the basal tachycardia seen in transplant recipients) and the rate response to exercise would be preserved. Whether this would achieve an improved exercise capacity after cardiac transplantation deserves further investigation. Osterholtzer et al ${ }^{12}$ report a 23 year old patient in whom sinus bradycardia after transplantation required pacemaker implantation. They describe the insertion of a dual chamber system with leads to recipient atrium (for sensing) and donor atrium (for sequential pacing). By setting the lowest delay $(65 \mathrm{~ms})$ they achieved physiological rate control using the recipient sinus node- "the most physiologic and best possible sensor". ${ }^{12}$ They do not present any haemodynamic data, however, and thus no conclusion can be drawn as far as any benefit from relative synchronism of contraction is concerned.

In conclusion, physiological pacing is better than ventricular pacing after transplantation. Synchronous donor-recipient atrial pacing confers no additional benefit at rest in these patients. Whether it confers a chronotropic advantage remains to be determined.

1 Miyamoto Y, Curtiss EI, Kormos RL, Armitage JM, Hardesty RL, Griffith BP. Bradyarrhythmia after heart
transplantation. Circulation 1990;82(suppl IV):IV-313-7. 2 Omar I, Scott CD, McComb JM, Dark JH, Bexton RS

Permanent pacing in heart transplant recipients [abstract] Permanent pacing in heart transplant recipient
Schweiz Med Wochenschr 1991:(suppl 42):12.

3 Heinz G, Ohner T, Laufer G, Gasic S, Laczkovics A.
Clinical and electrophysiologic correlates of sinus node dysfunction after orthotopic heart transplantation. Chest 1990;97:890-5.

4 Lascault G, Bogonzi F, Frank R, et al. Non-invasive study of dual chamber pacing by pulsed Doppler. Prediction of the haemodynamic response by echocardiographic measurements. Eur Heart $J 1989 ; 10: 525-31$.

5 Haskell RJ, French WJ. Physiological importance of different atrioventricular intervals to improved exercise performance in patients with dual chamber pacemakers. $\mathrm{Br}$ mance in patients with

6 Mehta D, Gilmour S, Ward DE, Camm AJ. Optimal atrioventricular delay at rest and during exercise in atrioventricular delay at rest and during exercise in patients with dual chamber pacemakers: a non-invasive
assessment by continuous wave Doppler. Br Heart J 1989; 61:161-6.

7 Cass MH, Brock R. Heart excision and replacement. Guy's Hospital Reports 1959;108:285-90

8 Valantine HA, Appleton CP, Hatle LK, Hunt SA, Stinson EB, Popp RL. Influence of recipient atrial contraction on left ventricular filling dynamics of the transplanted heart assessed by Doppler echocardiography. Am J Cardiol 1987;59:1159-63.

9 Clarke M, Sutton R, Ward D, et al. Recommendations for pacemaker prescription for symptomatic bradycardia. $\mathrm{Br}$ Heart J 1991;66:185-91.

10 Sutton R, Perrins E, Morley C, Chan S. Sustained improvement in exercise tolerance following physiological cardiac pacing. Eur Heart J 1983;4:781-5.

11 Jones RI, Cashman PMM, Hornung RS, Prince H, Bassein L, Raftery EB. Ambulatory blood pressure and assessL, Raftery EB. Ambulatory blood pressure and assess-

12 Osterholtzer G, Markewitz A, Anthumber M, Kemkes BM. An example of how to pace a patient with a heart An example of how to pace a patient with
transplantation. J Heart Transplant 1988;7:23-5.

13 Robson SC, Dunlop W', Moore M, Hunter S. Combined Robson SC, Dunlop W, Moore M, Hunter S. Combined
Doppler and echocardiographic measurement of cardiac output: Theory and application in pregnancy. $\mathrm{Br} \mathrm{JObstet}$ Gynaecol 1987;94:1028-39.

14 Goldberg SJ, Allen HD, Marx GR, Flinn CJ. Doppler Echocardiography. Philadelphia: Lea and Febiger, 1985: 68-91.

15 Midei MG, Baughman KL, Achuff SC, Walford GD, Baumgartner W, Brinker JA. Is atrial activation beneficial in heart transplant recipients? J Am Coll Cardiol 1990;16: 1201-4.

16 Gamong WF. Review of medical physiology. 7th ed. Los Altos: Lange, 1975:419-20.

17 Robson SC, Furniss SS, Heads A, Boys RJ, McGregor C, Bexton RS. Isometric exercise in the denervated heart: a Bexton RS. Isometric exercise in the denervated heart: a 224-30.

18 Pope SE, Stinson EB, Daughters GT, Schroeder JS, Ingels NB, Alderman EL. Exercise response of the denervated heart in long term cardiac transplant recipients. $\mathrm{Am} \mathrm{J}$ Cardiol 1980;46:213-8.

19 Bexton RS, Milne JR, Cory-Pearce R, English TAH, Camm AJ. Effect of beta blockade on exercise response after cardiac transplantation. Br Heart J 1983;49:584-8. 